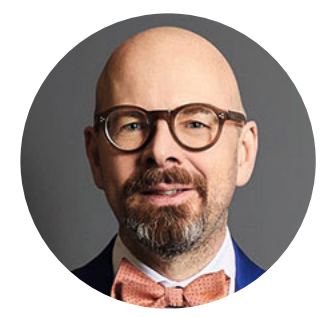

Jörg-Alexander Paul, Partner, Bird \& Bird

\section{Diskriminierungsfalle Künstliche Intelligenz: So vermeiden Sie Fehler schon beim Anlernen}

Um auch zukünftig wettbewerbsfähig zu bleiben, arbeiten viele Unternehmen bereits mit künstlicher Intelligenz. Viele Firmen trauen sich jedoch nicht an die vielversprechende Technologie ran, denn aktuelle Skandale rund um künstliche Intelligenz lassen vermuten: Die Innovation hat Konfliktpotenzial und kann schnell diskriminierend agieren.

Laut Presseberichten musste Amazon beispielsweise erst kürzlich eine auf künstlicher Intelligenz basierende HR-Software einstellen, denn diese benachteiligte Frauen im Bewerbungsprozess. Auch IBM sah sich nach solchen Berichten veranlasst, eine Gesichtserkennungssoftware vom Markt zu nehmen. Der Grund: Das Programm hatte Schwierigkeiten schwarze Personen zu identifizieren. Egal ob auf Grundlage von Geschlecht, Hautfarbe, Körperform oder Religion, die Beispiele für Diskriminierungen durch künstliche Intelligenz sind zahlreich. Was können Unternehmen also dafür tun, dass es gar nicht erst zu Diskriminierungen kommt?

\section{Die Gefahr erkennen}

Diskriminierungen können in verschiedenen Lebensphasen der künstlichen Intelligenz auftreten. Am häufigsten entstehen sie jedoch bereits beim Anlernen der Anwendung. So wird die künstliche Intelligenz schon ganz am Anfang ungewollt dazu veranlasst, diskriminierende Entscheidungen zu treffen. Selbst wenn die künstliche Intelligenz korrekt aufgesetzt ist, kann sie im Laufe ihres Lebenszyklus Muster erlernen, die ebenfalls in diskriminierendem Verhalten enden können.

Entstehen schon beim Anlernen „Fehler“ ist dies jedoch besonders ärgerlich, denn der Grund dafür ist oft - entgegen der schnell getroffenen Annahme nicht die unzureichende Qualität der eingepflegten Daten oder ein fehlerhafter Algorithmus - dass Programmierer und Programmiererinnen zu wenig divers bei der Datenauswahl und -bewertung denken.

\section{Divers denken beim Programmieren}

Die Vergangenheit zeigt, dass mangelnde Diversität beim Programmieren häufig zu diskriminierenden Anwendungen führt. Denn ohne diverses Denken beim Anlernen werden im Vorfeld nicht genügend Anwendungsszenarien durchdacht. Ein bekanntes, jedoch rein technisches Beispiel hierfür sind automatisierte Seifenspender eines bestimmten Typs. Diese konnten keine schwarze Hautfarbe erkennen. Der Grund: Die Programmierer der dazu notwendigen Infrarot-Standard-Technologie waren überwiegend kaukasischen Typs und haben die Nutzung von Seifenspendern durch dunkelhäutige Personen nicht bedacht.
Ebenso häufig entstehen Diskriminierungen, weil beim Anlernen der künstlichen Intelligenz auf rein mathematische Aspekte geachtet wird. Bei einer KI-basierten Bewerbungsplattform, könnte der einprogrammierte Algorithmus beispielsweise mathematisch zu der Auswertung kommen, dass Männer für die Produktivität eines Unternehmens wertvoller sind als Frauen - zumindest, wenn er sie an ihren Fehltagen misst. Denn Frauen haben statistisch aufgrund diverser Faktoren, wie Schwangerschaften oder der tradierten Kinderfürsorge, mehr Fehlzeiten als Männer. Normative Korrekturen müssen daher schon im Vorfeld beim Anlernen der künstlichen Intelligenz wortwörtlich einprogrammiert werden.

\section{Der künstlichen Intelligenz Werte beibringen}

Die HR-Software zeigt: KI-Anwendungen arbeiten neutral. Sie erkennen Muster und schlussfolgern basierend auf diesen. Entscheidungen von künstlicher Intelligenz reflektieren daher nicht unbedingt die Werte, auf die wir uns innerhalb unserer Gesellschaft geeinigt haben. Schon beim Anlernen der KI-Anwendung sollte daher reflektiert werden, welche - möglicherweise ungewünschten - Schlüsse die KI aus den eingefügten Daten ziehen könnte und welches Ergebnis eigentlich gewünscht wäre.

Den Fehler, dass künstliche Intelligenz aufgrund von Daten ungewollte Rückschlüsse zieht, machte nach den Medien 2016 auch Microsoft. Das Unternehmen hatte einen digitalen Schönheitswettbewerb mitorganisiert, der auf künstlicher Intelligenz basierte. Ziel war es, mithilfe eines Algorithmus und 600.000 eingesendeten Selfies die „schönste“ Person zu küren. Am Ende befand sich unter den 44 gewählten Gewinnern und Gewinnerinnen jedoch nur eine dunkelhäutige Person - was zu Empörung führte. Der Grund dafür war, dass der Algorithmus mit einer Datenbank trainiert wurde, die überwiegend aus Bildern besteht, die Menschen mit kaukasischem Typ abbilden.

Für beide Beispiele gilt: In die Algorithmen, nach denen künstliche Intelligenz arbeitet, müssen normative Werte eingebaut werden, z.B.: Frauen sind wertvolle Mitarbeiterinnen, auch wenn sie statistisch gesehen mehr Fehltage haben als Männer. Ebenso sollte im Vorfeld darüber nachgedacht werden, welche Rückschlüsse die KI aus überwiegend männlichen Bewerbungen ziehen könnte. Es sollte hier vorher definiert werden, dass das Geschlecht kein Maßstab für die Qualifikation eines Bewerbers oder einer Bewerberin ist. Heiß diskutiert wird diese Thematik auch unter Juristen und dem Staat, denn der greift bisher - zumindest beim Anlernen - gar nicht ein.

\section{Keine Regulierungen beim Anlernen der KI \\ Derzeit geben lediglich unverbindliche Ethik-Leit- linien eine Orientierung beim Anlernen von künst- licher Intelligenz. Führt die Anwendung allerdings}


diskriminierende Handlungen aus, drohen rechtliche Konsequenzen. Auch gibt es rechtliche Rahmenbedingungen, die sich aus den allgemeinen Vorschriften ergeben und unbedingt beachtet werden sollten. Beispielsweise kann es Dokumentationspflichten beim Anlernen geben, die erfüllt werden müssen. Werden diese missachtet, kann es sein, dass das Endprodukt nicht angewendet werden darf.

Die aktuelle Rechtslage ist sehr liberal und kommt der Innovation von Unternehmen zu Gute, denn diese können an der künstlichen Intelligenz arbeiten, ohne sich bereits beim Anlernen einer Vielzahl von hemmenden Regularien zu unterwerfen. Dennoch diskutiert der Gesetzgeber derzeit, rechtlich einzugreifen, denn die einprogrammierten Diskriminierungen sind im Nachhinein häufig nur schwer zu erkennen. Dass Amazons Bewerber-Software diskriminierend gegenüber Frauen agierte, soll den Entwicklern beispielsweise erst nach einem Jahr aufgefallen sein.

\section{Trauen Sie sich an künstliche Intelligenz!}

Deutsche Unternehmen müssen sich mit Innovationen wie künstlicher Intelligenz auseinandersetzen, um kompetitiv zu bleiben. Ohnehin begünstigt die aktuelle Rechtslage den Innovationsspielraum von Unternehmen.

Nutzen Sie dies, achten Sie jedoch darauf, sich an die ethische Leitlinien zu halten und sich schon beim Anlernen Gedanken darüber zu machen, wie divers das Daten-Set ist, wer die Anwendung benutzen könnte (auch außerhalb des eigenen Typs) und welche unerwünschten Rückschlüsse die KI aus den eingepflegten Daten ziehen könnte. So vermeiden Sie Diskriminierungen und im Zweifel auch staatliches Eingreifen und Angriffe von Konkurrenten.

Jörg-Alexander Paul

\subsection{MEDIZIN}

\section{Eine zweite Chance: Wie sich Unternehmen besser auf die nächste COVID-19-Welle vorbereiten können}

Mittlerweile ist mehr als ein halbes Jahr seit Beginn der Corona-Pandemie vergangen. In vielen Ländern Europas wurden bereits vor über sechs Monaten weitreichende Notfallmaßnahmen eingeführt, um gegen die Verbreitung von COVID-19 vorzugehen. Die Auswirkungen, die das neuartige Virus auf die Gesellschaft hat, sind immens - sowohl in humanitärer als auch in geschäftlicher Hinsicht.

Die Pandemie hat zu erheblichen Einnahme- und Produktivitätseinbußen von Unternehmen verschie- denster Branchen geführt. In der Folge gingen tausende Arbeitsplätze verloren und wir stehen vor einer weltweiten Rezension. Auch die Art und Weise hat sich geändert, wie Unternehmen ihre Lieferketten strukturieren, planen und verwalten. Das Konzept statischer Netzwerke gehört der Vergangenheit an, dynamische Wertschöpfungsketten sind ein Muss.

In letzter Zeit gab es sicherlich Anzeichen einer Erholung der globalen Lieferketen, bedingt durch die Lockerung der Notfallmaßnahmen und das Umdenken von Unternehmen in Bezug auf deren Lieferketten. Dennoch bleibt eine gewisse Unsicherheit - speziell im Hinblick auf eine zweite oder sogar dritte Welle der weltweiten COVID-19-Infektionen. Um auf alle Eventualitäten vorbereitet zu sein, müssen Unternehmen die Erfahrungen der letzten Monate nutzen, sodass sie nicht erneut unvorbereitet getroffen werden.

\section{„Beispiellos" war gestern}

Als COVID-19 erstmals die Ausmaße einer globalen Pandemie angenommen hat, wurde sie oftmals als „beispiellos“ bezeichnet. Niemand konnte die Pandemie voraussehen und vergleichbare Szenarien in der Menschheitsgeschichte gab es nur wenige. Die WHO hat sie sogar als den "schwersten" globalen Gesundheitsnotstand aller Zeiten bezeichnet. Die Zeiten sind jedoch vorbei, in denen man von einer beispiellosen Ausnahmesituation sprechen kann. Die erste Welle hat mehr als deutlich gemacht, mit welchen Auswirkungen die Gesellschaft und die Industrie infolge einer zweiten Welle rechnen muss. Der Präzedenzfall steht und muss genutzt werden, um in gesellschaftlicher und wirtschaftlicher Hinsicht widerstandsfähiger und agiler reagieren zu können, als noch zu Beginn der Krise. Wir haben nun lange genug mit dem Virus gelebt und wissen, welche Störungen es verursacht, mit welchen $\mathrm{Maßnahmen} \mathrm{wir} \mathrm{diesen} \mathrm{erfolgreich} \mathrm{begegnen} \mathrm{und} \mathrm{wie}$ wir sie schnellstmöglich umsetzen können.

Seien es Hamsterkäufe und die daraus resultierende Verknappung von Konsumgütern wie Toilettenpapier oder das Problem von Überbeständen infolge schwankender Produktnachfrage - es liegen ausführliche Daten über die Auswirkungen von COVID-19 auf die verschiedensten Bereiche vor. Trifft uns die nächste Infektionswelle, verfügen Unternehmen über die nötigen Informationen und dank künstlicher Intelligenz und maschinellem Lernen auch über die adäquaten technologischen Hilfsmittel, um sich richtig vorzubereiten und schnell zu reagieren.

\section{Lektion gelernt}

Durch einen Rückblick auf die letzten sechs Monate, können Unternehmen eine Vielzahl wichtiger Schlüsse ziehen. Sie können evaluieren, was schiefgelaufen ist und dafür sorgen, dass sich die gemachten Fehler nicht wiederholen. Eines der größten Probleme im Zuge der Pandemie war für viele Unternehmen der umfassende

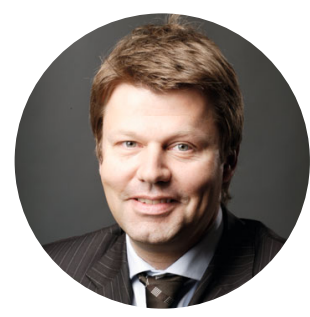

Michael Wallraven, Geschäftsführer und Regional

Vice President, LLamasoft Deutschland GmbH 